\title{
Síndrome de Satoyoshi: Enfermedad multisistémica con respuesta exitosa a tratamiento esteroidal
}

\author{
Claudia Castiglioni ${ }^{1,3}$, Alejandra Díaz $^{1}$, Karla Moënne $^{2}$, \\ Verónica Mericq ${ }^{3}$, Fernando Salvador ${ }^{1}$, Carolina Hernández ${ }^{1}$. \\ Satoyoshi syndrome. \\ Report of one case
}

Satoyoshi syndrome is a rare multisystemic disease of presumed autoimmune etiology characterized by progressive painful intermittent muscle spasms, diarrhea frequently associated with malabsorption, alopecia, skeletal abnormalities and endocrine disorders with a poor long-term prognosis due to early crippling. We report a 14-year-old Chilean girl with clinical and radiological features of the syndrome who has been successfully treated with prednisone and carbamazepine. She remarkably recovered from muscle spasms, alopecia and diarrhea. At follow up, 24 months later, she persists asymptomatic with considerable improvement in her quality of life (Rev Méd Chile 2009; 137: 542-6).

(Key-words: Carbamazepine; Satoyoshi syndrome; Spasm)

Recibido el 3 de julio, 2008. Aceptado el 10 de octubre, 2008.

${ }^{1}$ Instituto Nacional de Rehabilitación Pedro Aguirre Cerda. ${ }^{2}$ Departamentos de Radiología y ${ }^{3}$ Pediatría Clínica Las Condes. Santiago de Chile.

\begin{abstract}
$E^{1}$ síndrome de Satoyoshi es una enfermedad multisistémica descrita por Satoyoshi y Yama$\mathrm{da}^{1}$ en 1967. Se caracteriza por la presencia de espasmos musculares dolorosos, diarrea, alopecia y alteraciones endocrinológicas ${ }^{1,2}$. Presenta además compromiso esquelético que incluye lesiones osteolíticas a nivel de las epífisis y metáfisis de huesos largos ${ }^{3-5}$ atribuidas al daño sobre el cartílago de crecimiento, epífisis y zonas de inserción tendinosa secundarias a los vigorosos y repetidos espasmos musculares ${ }^{2,4}$. En la literatura se han comunicado alrededor de 52 casos. Hallazgos
\end{abstract}

Correspondencia a: Claudia Castiglioni. Lo Fontecilla 441 Las Condes. Fax: 6108413. E-mail: ccastiglioni@clc.cl recientes ${ }^{6}$, describen la presencia de anticuerpos anti-lisados de tejido cerebral, estómago y duodeno humanos, que sustentan la sospecha de un origen autoinmune para este síndrome multisistémico. También se ha comunicado su asociación con otras enfermedades autoinmunes como miastenia gravis 7 , púrpura trombocitopénico idiopáti$\mathrm{Co}^{6}$ y se ha descrito en algunos pacientes la presencia en sangre de anticuerpos antinucleares y antiADN ${ }^{8}$, antirreceptores de acetilcolina ${ }^{9}$, y antiGAD (decarboxilasa del ácido glutámico) ${ }^{10}$.

La edad de inicio de la enfermedad fluctúa entre los 5 y 19 años ${ }^{6,11}$, existiendo también casos de presentación en adultos ${ }^{12,13}$. La gran mayoría han sido descritos en Japón, afectando a las mujeres en una proporción casi el doble que los hombres ${ }^{11}$. 
Comunicamos el primer caso descrito en Chile de síndrome de Satoyoshi en una niña de 14 años de edad, con respuesta exitosa al tratamiento esteroidal y carbamazepina, con una revisión de la literatura pertinente.

\section{Caso Cúnico}

Paciente de sexo femenino de 14 años, hija de padres no consanguíneos, la menor de 5 hermanos sanos. Sin antecedentes relevantes perinatales con peso de nacimiento de $3,860 \mathrm{gr}$ y talla de 52 $\mathrm{cm}$. Evolucionó con un desarrollo psicomotor normal; escolarizada de acuerdo a su edad.

El cuadro clínico se inició insidiosamente a los 9 años de edad con episodios recurrentes de dolor abdominal y lumbar, asociados en forma progresiva a espasmos musculares involuntarios de extremidades, tronco, cuello y cara, llegando en varios meses a presentar numerosos episodios diarios de aproximadamente 2 a 3 min de duración, en reposo, actividad, vigilia y durante el sueño. Aproximadamente al año de evolución consultó en su hospital de origen por diarrea intermitente, periódica, con deposiciones líquidas de hasta 10 veces por día, acompañada de compromiso pondo estatural y progresiva caída del cabello. Dos años posterior al inicio de estos síntomas se instaló una deformidad en varo de ambas rodillas (Figura 1A) muy dolorosa que limitaba la marcha, lo que motivó una intervención quirúrgica de su rodilla derecha en 2 oportunidades.

A los 12 años, fue referida a nuestro centro, con una talla de $133 \mathrm{~cm}$ y un peso de $28,5 \mathrm{~kg}$ ambas medidas inferiores a 3 desviaciones estándar para su edad. Su índice de masa corporal era $16,1 \mathrm{~kg} / \mathrm{m}^{2}$; percentil 15 de la tabla de referencia ${ }^{14}$, con una proporción normal de segmentos corporales. Su desarrollo puberal mamario y pubiano correspondía a Tanner I. Destacaba su cabello ralo con extensas zonas de alopecia en regiones frontoparietales y una hipertrofia de ambos músculos maseteros (Figuras 1B, C). Se observaron espasmos musculares frecuentes en extremidades durante el examen físico que ocasionaban en algunas oportunidades posturas anómalas de ellas. El hemograma, bioquímica hepática y renal, electrolitos, calcio y fósforo en sangre fueron normales. La creatinkinasa total en sangre estaba elevada a $290 \mathrm{UI} / \mathrm{l}(\mathrm{VN}<174)$.

La historia clínica hizo revisar las múltiples radiografías esqueléticas de la paciente por uno de los autores, las cuales mostraban alteraciones metafisiarias de distinta severidad en los distintos segmentos óseos, asimétricas, consistentes en lesiones líticas y áreas de esclerosis que comúnmente se observan en el síndrome de Satoyoshi, localizadas a nivel de manos, fémur distal y tibia distal (Figura 2).

Una electromiografía y velocidad de conducción nerviosa no mostró alteraciones, y una

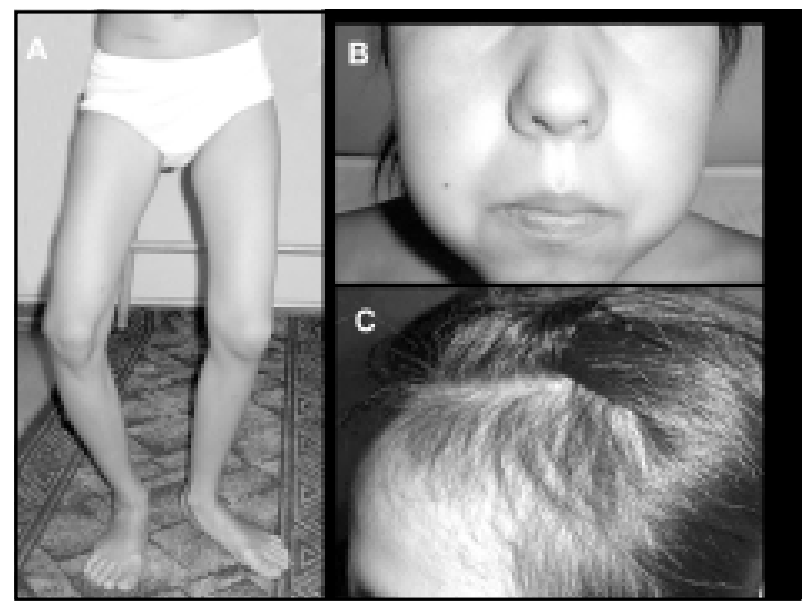

Figura 1. A. deformación en varo de ambas rodillas. B. hipertrofia de músculos maseteros. C. segmentos alopécicos en regiones frontales de cuero cabelludo. 


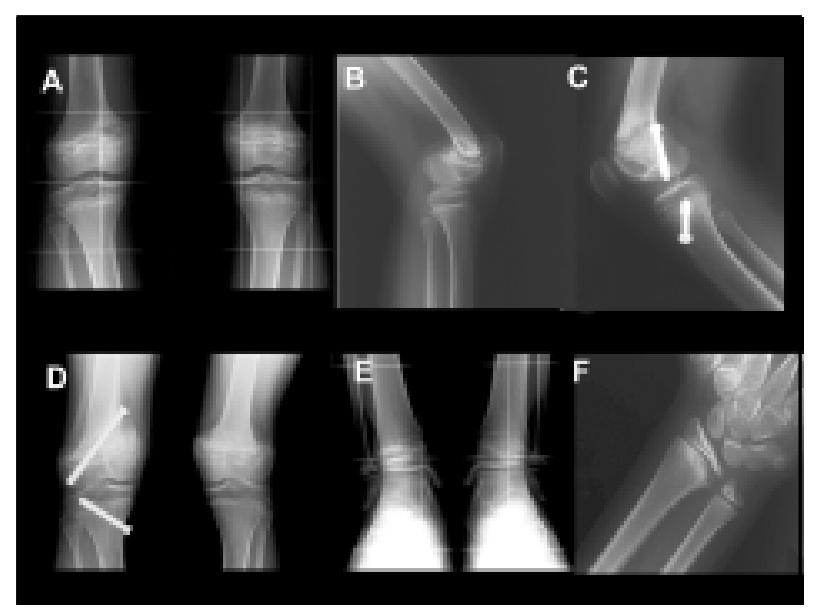

Figura 2. Radiografías (A-D rodillas; E tobillo; F muñeca) muestran alteraciones metafisiarias de severidad variable en los distintos segmentos, consistentes en lesiones líticas y áreas de esclerosis. A nivel de rodillas (AD) se observa evolución de lesiones en años sucesivos, con aparición de fractura y posterior osteosíntesis.

resonancia magnética medular reveló una pequeña siringohidromielia a nivel de los segmentos dorsales T3 a T7, sin expresión clínica.

El estudio de la diarrea comprendió un test de D- Xilosa que demostró una disminución de la excreción de xilosa en orina, 2,39 g/5 hr (VN: $\geq 4$ gr/ $5 \mathrm{hr}$ ), concordante con una malabsorbción. En la endoscopia digestiva alta se observaron leucoplaquias abundantes en el duodeno y una vasculatura pronunciada, con un estudio histológico de la mucosa que describe un aumento moderado del infiltrado linfoplasmocitario de la lámina propia con granulocitos neutrófilos y eosinófilos.

Los anticuerpos antiendomisio y el estudio de sangre oculta en deposiciones (test de Guayaco sensibilizado) fueron negativos.

El estudio endocrinológico fue compatible con un hipogonadismo hipergonadotrófico sugerente de una falla ovárica.

Se inició terapia corticoidal con prednisona oral 1,5 mg/ $\mathrm{kg} /$ día por 10 días, observándose en la primera semana de tratamiento una importante reducción en severidad y frecuencia de los espasmos musculares dolorosos que desaparecieron completamente al agregar carbamazepina $200 \mathrm{mg} /$ día. La diarrea, si bien disminuyó con el tratamiento esteroidal, no mejoró significativamente hasta el 5 mes cuando se indicó incorporar una estricta dieta con restricción de carbohidratos simples.
Su seguimiento a 2 años (edad: 14 años, 3 meses, desarrollo puberal Tanner II), con prednisona en días alternos en dosis decrecientes ha constatado ausencia de espasmos musculares, la desaparición de la alopecia y la diarrea, un incremento en el peso que se había mantenido estacionario los últimos tres años, de 35,800 kg, si bien persiste con una baja estatura y con las deformidades esqueléticas residuales a nivel de rodillas.

\section{DISCUSIÓN}

A nuestro conocimiento este es el primer caso chileno comunicado de Síndrome de Satoyoshi. Esta paciente presenta el cuadro clínico completo ${ }^{1,2}$, siendo el segundo caso descrito en Sudamérica ${ }^{12}$. La otra comunicación es de un varón argentino adulto con espasmos fundamentalmente de los músculos de la masticación que le ocasionaban trismus severos y dificultades alimentarias secundarias. Recibió toxina botulínica para la manifestación local de la enfermedad ${ }^{15}$. Llamativamente no se indicaron corticoides, lo que puede ser explicado porque los primeros artículos sobre una patogénesis autoinmune están en literatura en lengua japonesa y datan de 1991 y 1992.

Nuestra paciente inició sus síntomas en período prepuberal con un retardo del diagnóstico de 3 
años que ocasionó un compromiso esquelético importante y un daño irreversible sobre las metáfisis femorales con la consiguiente repercusión sobre la talla final. Este caso, similar a otros ${ }^{5,15}$, muestra la importancia de establecer el diagnóstico en forma oportuna, dado que el tratamiento esteroidal, permite evitar estas graves consecuencias en los pacientes que inician su enfermedad en edad pediátrica.

Los espasmos musculares involuntarios, cuya relación con el probable origen autoinmune del síndrome no está aclarada, parecen ser secundarios a una desregulación de la motoneurona a nivel del asta anterior de la médula espinal, según sugieren autores en base a estudios electromiográficos ${ }^{16}$. Se han utilizado para tratarlos, dantrolen, benzodiacepínicos, anticonvulsivantes y toxina botulínica $^{2,12}$ con resultados variables y que no están enfocados a la patogenia de la enfermedad. Desde los años noventa la mayoría de los pacientes con síndrome de Satoyoshi se han beneficiado del tratamiento con corticoides que se ha mantenido por más de un año $0^{5,11}$, con una significativa reducción de los espasmos musculares, revirtiendo la caída del cabello y logrando el inicio de la menstruación en algunos casos de mujeres afectadas $5^{5,7-9,11,15,17-20}$. También se han utilizado inmunosupresores como metotrexato, tacrolimus e inmunoglobulinas ${ }^{9,11}$ que pueden ser alternativas útiles cuando las dosis de esteroides necesarias para revertir los síntomas ocasionan efectos adversos indeseables o respuesta parcial.

La diarrea que se observa en más de la mitad de los casos, en ocasiones de muy difícil manejo, podría ser secundaria también a un mecanismo autoinmune ${ }^{6}$. En nuestra paciente, los hallazgos

\section{REFERENCIAS}

1. Satoyoshi E, Yamada K. Recurrent muscle spasms of central origin: a report of two cases. Arch Neurol 1967; 16: 254-64.

2. Satoyoshi E. A syndrome of progressive muscle spasm, alopecia, and diarrhea. Neurology 1978; 28: 458-71.

3. Matsuo N, Fujioka M, Tsuchiya $\mathrm{Y}$, Cho H, Nagai T, Kumagai M. Multiple metaphyseal lesions in a child with a syndrome of progressive muscle cramps, alopecia and stunted growth (Satoyoshi disease). Radiat Med 1983; 1: 205-7. en la biopsia duodenal sugerentes de malabsorción e infiltración inflamatoria de la mucosa concuerdan con los descritos en una mujer de 17 años portadora de este síndrome ${ }^{6}$. El manejo de la dieta con restricción estricta de carbohidratos simples (fructosa, sacarosa, lactosa) fue un factor determinante en el control de la diarrea. El retraso en el diagnóstico y tratamiento apropiado puede conducir a una emaciación generalizada por compromiso nutricional secundario a la malabsonción y a dificultades en la alimentación secundarias a espasmos de los músculos masticatorios.

El retraso puberal y la presencia de amenorrea ocurre casi en la totalidad de las pacientes ${ }^{2,5,6,8-}$ 11,13,14. En nuestro caso, el retraso puberal presentaba niveles elevados de gonadotrofinas basales con bajos niveles de estradiol, lo que indica un hipogonadismo hipergonadotropo, planteándose la posibilidad de una ooforitis autoinmune como mecanismo responsable de estos trastornos endocrinológicos. En la citada comunicación ${ }^{6}$ que mostró la presencia de anticuerpos anti tejido cerebral, útero, estómago y duodeno, desafortunadamente no se estudió el tejido ovárico. La estimulación con hormona liberadora de gonadotropinas ( $\mathrm{GnRH})$ no fue realizada de manera rutinaria en los estudios previos, con el fin de diferenciar entre hipogonadismo hipo o hiper-gonadotrófico.

Genetistas, neurólogos, endocrinólogos, gastroenterólogos, ortopedistas, dermatólogos, radiólogos y pediatras deben conocer esta enfermedad multisistémica, que, pese a ser muy poco frecuente, está descrita en todos los grupos étnicos. Sólo su correcto diagnóstico permitirá una oportuna intervención terapéutica que logre revertir los síntomas y así mejorar la calidad de vida.

4. Ikegawa S, Nagano A, Satoyoshi E. Skeletal abnormalities in Satoyoshi syndrome: a radiographic stydy of eight cases. Skeletal Radiol 1993; 22: 321-24.

5. Haymon M, Wiws R, Ehlayel M, Lacassie Y. Radiological and orthopedic abnormalities in Satoyoshi syndrome. Pediatr Radiol 1997; 27: 415-8.

6. Matsuura E, Matsuyama W, Sameshima T, Arimura K. Satoyoshi syndrome has antibody against brain and gastrointestinal tissue. Muscle Nerve 2007; 36: 400-3.

7. Satoh A, Tsujihata M, Yoshimura T, Mori M, Nagataki S. Myasthenia gravis associated with Satoyoshi syndrome: muscle cramps, alopecia, and diarrhea. Neurology 1983; 33: 1209-11. 
8. Arita J, Hamano S, Nara T, Maekawa K. Intravenous gammaglobulin therapy of Satoyoshi syndrome. Brain Dev 1996; 18: 409-11.

9. Endo K, Yamamoto T, NaKAmURa $K$, Hoshi A, Yamano T, Watanabe A, ET AL. Improvement of Satoyoshi syndrome with tacrolimus and corticosteroids. Neurology 2003; 60: 2014-5.

10. Drost G, Verrips A, Hooijkaas H, Zwarts M. Glutamic acid decarboxylase antibodies in Satoyoshi syndrome. Ann Neurol 2004; 55: 450-1.

11. Heger S, Kuester R, Volk R, Stephani U, Sippell W. Satoyoshi syndrome: A rare multisystemic disorder requiring systemic and symptomatic treatment. Brain Dev 2006; 28: 300-4.

12. Merelo M, García $H$, Nogues M, Leiguarda R. Masticatory muscle spasm in a non-Japanese patient with Satoyoshi syndrome successfully treated with botulinum toxin. Mov Disord 1994; 9: 104-5.

13. IKeda K, Satoyoshi E, Kinoshita M, WaKata N, IwaSAKI Y. Satoyoshi's syndrome in an adult: A review of the literature of adult onset cases. Intern Med 1998; 37: 784-7.
14. Kuczmarski R, Ogden C, Grummer-Strawn L CDC Growth Charts: United States. Advanced data from vital and health statistics; № 314. Hyattsville, Maryland: Editorial National Center for Health Statistics 2000.

15. Ehlayel M, Lacassie Y. Satoyoshi syndrome: an unusual posnatal multisystemic disorder. Am J Med Genet 1995; 57: 620-5.

16. Drost G, Verrips A, Van Engelen B, Stegeman D, Zwarts M. Involuntary Painful Muscle Contractions in Satoyoshi Syndrome: A Surface Electromyographic Study. Mov Disord 2006; 21: 2015-8.

17. Ashalatha R, Kishore A, Sarada C, Nair M. Satoyoshi syndrome. Neurol India 2004; 52: 94-5.

18. Cecchin C, Felix T, Magalhaes R, Furlanetto T. Satoyoshi syndrome in a caucasian girl improved with glucocorticoids. Am J Med Genet 2003; 118: 52-4.

19. Oyama M, Imaizumi T, Mitsuhashi $Y$, Kondo S. Satoyoshi syndrome. Arch Dermatol 1999; 135: 91-2.

20. Kamat D, Petry L, BerRy S. A case of Satoyoshi syndrome: a multisystemic disorder. Clin Pediatr 2003; 42: 745-8. 